\title{
Exact Static Soliton Solutions of $(3+1)$-Dimensional Integrable Theory with Nonzero Hopf Numbers
}

\author{
H. Aratyn, ${ }^{1}$ L. A. Ferreira, ${ }^{2}$ and A. H. Zimerman ${ }^{2}$ \\ ${ }^{1}$ Department of Physics, University of Illinois at Chicago, 845 W. Taylor Street, Chicago, Illinois 60607-7059 \\ ${ }^{2}$ Instituto de Física Teórica-IFT/UNESP, Rua Pamplona 145, 01405-900, São Paulo-SP, Brazil
}

(Received 17 May 1999)

In this paper, we explicitly construct an infinite number of Hopfions (static, soliton solutions with nonzero Hopf topological charges) within the recently proposed $(3+1)$-dimensional, integrable, and relativistically invariant field theory. Two integers label the family of Hopfions we have found. Their product is equal to the Hopf charge which provides a lower bound to the soliton's finite energy. The Hopfions are explicitly constructed in terms of the toroidal coordinates and shown to have a form of linked closed vortices.

PACS numbers: 11.10.Lm, 05.45.Yv, 11.27.+d

Recent numerical studies of the Faddeev-Skyrme modified $O(3)$ sigma model [1] support the existence of static toroidal solutions stabilized by their nonzero Hopf numbers [2]. The emerging stringlike structures are quite intriguing and may find applications in various physical models of condensed matter physics and gauge field theory. It is therefore of direct physical interest to find a field theoretical model for which it is possible to write down in a closed form explicit soliton solutions with nonzero Hopf index (Hopfians). This will advance an understanding of stringlike soliton configurations and their properties and open a way to incorporate them into various models relevant for physical applications.

In Ref. [3], we have introduced the three-dimensional field model which falls into a class of higher dimensional integrable models from the point of view of the generalized zero-curvature approach [4]. The question posed in [3] was whether this form of integrability is linked to the existence of soliton solutions as is expected from the study of two-dimensional integrable models. Our analysis of the model in [3] has indeed revealed one nontrivial soliton solution described by a standard Hopf map of unit Hopf index. To fully establish a connection between integrability and soliton solutions would require finding other topological solitons with arbitrary topological charges. This is accomplished in this Letter. The equations of motion of the model are solved in toroidal coordinates and the space of solutions is found to be represented by a family of maps $\mathbb{R}^{3} \rightarrow \mathbb{R}^{2}$ labeled by two integers. The integers count the number of times the map winds around two independent angular directions.

The model under consideration is described by the Lagrangian density

$$
\mathcal{L}=-\eta_{0}\left(H_{\mu \nu}^{2}\right)^{3 / 4},
$$

where $\eta_{0}= \pm 1$ determines the choice of the signature of the Minkowski metric, $g_{\mu \nu}=\eta_{0} \operatorname{diag}(1,-1,-1,-1)$, and the field tensor $H_{\mu \nu}$ is defined in terms of the three component, unit vector field $\vec{n} \in S^{2}$ as

$$
H_{\mu \nu} \equiv \vec{n} \cdot\left(\partial_{\mu} \vec{n} \times \partial_{\nu} \vec{n}\right) .
$$

The action in (1) is $\mathrm{O}(3)$ and Poincare invariant. The value $\frac{3}{4}$ of the power of $H_{\mu \nu}^{2}$ in (1) is such that the theory circumvents the usual obstacle of Derrick's scaling argument against the existence of stable solitons. We are interested in the boundary condition $\vec{n}=(0,0,1)$ at spatial infinity. This condition compactifies effectively the Euclidean space $\mathbb{R}^{3}$ to the three-sphere $S^{3}$. Accordingly, $\vec{n}$ becomes a map: $S^{3} \rightarrow S^{2}$. Because of $\pi_{3}\left(S^{2}\right)=\mathbb{Z}$, the field configurations fall into disjoint classes characterized by the value of the Hopf invariant $Q_{H}$.

Using stereographic projection of $S^{2}$,

$$
\vec{n}=\frac{1}{1+|u|^{2}}\left(u+u^{*},-i\left(u-u^{*}\right),|u|^{2}-1\right),
$$

one obtains

$$
H_{\mu \nu}=\frac{-2 i}{\left(1+|u|^{2}\right)^{2}}\left(\partial_{\mu} u \partial_{\nu} u^{*}-\partial_{\nu} u \partial_{\mu} u^{*}\right),
$$

where $u$ is the complex scalar field.

In terms of the vector quantities

$$
\begin{aligned}
K_{\mu} & =\frac{i}{2}\left(1+|u|^{2}\right)^{2} H_{\mu \nu} \partial^{\nu} u \\
& =\left(\partial^{\nu} u^{*} \partial_{\nu} u\right) \partial_{\mu} u-\left(\partial_{\nu} u\right)^{2} \partial_{\mu} u^{*}
\end{aligned}
$$

and

$$
\mathcal{K}_{\mu} \equiv \frac{\left(K \partial u^{*}\right)^{-1 / 4} K_{\mu}}{1+|u|^{2}},
$$

the corresponding equations of motion can be rewritten compactly as

$$
\partial^{\mu} \mathcal{K}_{\mu}=0 .
$$

Notice that the Lagrangian density (1) has a branch cut when $H_{\mu \nu}$ vanishes. Both $K_{\mu}$ and $H_{\mu \nu}$ vanish for configurations of the form $u \equiv \rho(\theta) e^{i \theta}$, where $\rho$ is an arbitrary function of $\theta$, and $\theta$ is an arbitrary and real function of the space-time coordinates. In what follows we shall exclude such class of vacuum configurations. 
The quantity (5) automatically satisfies the relations

$$
K^{\mu} \partial_{\mu} u=0 \quad \operatorname{Im}\left(K^{\mu} \partial_{\mu} u^{*}\right)=0,
$$

which play a crucial role in establishing integrability of the model. Indeed, using relations (8) and the equations of motion (7) one obtains an infinite number of conserved currents given by

$$
J_{\mu} \equiv \mathcal{K}_{\mu} \frac{\delta G}{\delta u}-\mathcal{K}_{\mu}^{*} \frac{\delta G}{\delta u^{*}},
$$

with $G$ being any functional of $u$ and $u^{*}$ only (no derivatives). In Ref. [3] we have analyzed the integrability properties of this theory using the generalized version of the zero curvature condition [4]. The equations of motion (7) can be represented as $\partial^{\mu} B_{\mu}+\left[A^{\mu}, B_{\mu}\right]=0$, with $A^{\mu}$ being a flat $\mathrm{SU}(2)$ connection, and $B_{\mu}$ being an operator living in any integer spin representation of SU(2) (for more details see $[3,4]$ ). The integrability properties emerge due to the fact that $B_{\mu}$ can be put in any integer spin representation, and that is a direct consequence of Eq. (8) [4,5]. In Ref. [3], we have shown that the existence of the conserved currents as in (9) is related to "hidden symmetries" of the equations of motion. In other words, the zero curvature condition, which is equivalent to the equations of motion, is invariant under an infinite nonsemisimple gauge group, as a consequence of the existence of a singlet state of the integer spin representations of $\mathrm{SU}(2)$.

Another point is whether such an infinite number of conservation laws leads to the exact integrability of the model, in a way similar to what happens in the two-dimensional integrable theories. This question is addressed here indirectly by showing the existence of an infinite number of static soliton solutions.

What shall look for time-independent solutions to equations of motion (7) of the type (we shall take $m$ and $n$ integers in order for $u$ to be single valued)

$$
u(\eta, \xi, \varphi) \equiv f(\eta) e^{i(m \xi+n \varphi)},
$$

where we used toroidal coordinates on $\mathbb{R}^{3}$ :

$$
\begin{gathered}
x=a q^{-1} \sinh \eta \cos \varphi, \quad y=a q^{-1} \sinh \eta \sin \varphi, \\
z=a q^{-1} \sin \xi ; \quad a>0 ; \quad q=\cosh \eta-\cos \xi
\end{gathered}
$$

The angles $\xi, \varphi$ both vary from 0 to $2 \pi$ and $\eta$ varies from 0 to $\infty$. The surfaces of constant $\eta$ are toroids that circle the $z$ axis, $\xi=$ const are spheres, and $\varphi=$ const are half-planes. The corresponding gradient of $u$ becomes in toroidal coordinates

$$
\begin{aligned}
\vec{\nabla} u=(q / a) e^{i(m \xi+n \varphi)} & \left(f^{\prime}(\eta) \hat{e}_{\eta}+i m f(\eta) \hat{e}_{\xi}\right. \\
& \left.+\inf (\eta) \frac{\hat{e}_{\varphi}}{\sinh \eta}\right),
\end{aligned}
$$

where we introduced the unit vectors spanning the orthogonal toroidal coordinate system having properties $\hat{e}_{i} \cdot \hat{e}_{j}=\delta_{i j}, i, j \equiv \eta, \xi, \varphi$.
In terms of the vector-field $\overrightarrow{\mathcal{K}} \equiv(\vec{K}$. $\left.\vec{\nabla} u^{*}\right)^{-1 / 4} \vec{K} /\left(|u|^{2}+1\right)$ the equations of motion (7) become, in the static case, $\vec{\nabla} \cdot \overrightarrow{\mathcal{K}}=0$; i.e., $\overrightarrow{\mathcal{K}}$ is solenoidal. Using (10) and (12) we obtain for the components of $\overrightarrow{\mathcal{K}}$

$$
\begin{gathered}
\mathcal{K}_{\eta}=\mathcal{K}_{0}\left(m^{2}+\frac{n^{2}}{(\sinh \eta)^{2}}\right) f ; \quad \mathcal{K}_{\xi}=i m \mathcal{K}_{0} f^{\prime} \\
\mathcal{K}_{\varphi}=\operatorname{in}_{\mathcal{K}} \frac{f^{\prime}}{\sinh \eta},
\end{gathered}
$$

with

$$
\begin{aligned}
\mathcal{K}_{0} \equiv & \sqrt{2}(q / a)^{2} e^{i(m \xi+n \varphi)}\left(m^{2}+\frac{n^{2}}{(\sinh \eta)^{2}}\right)^{-1 / 4} \\
& \times \frac{f^{1 / 2} f^{\prime 1 / 2}}{\left(f^{2}+1\right)}
\end{aligned}
$$

where the components of the vector field are defined according to $\vec{V}=\hat{e}_{\eta} V_{\eta}+\hat{e}_{\xi} V_{\xi}+\hat{e}_{\varphi} V_{\varphi}$.

Plugging the components of $\overrightarrow{\mathcal{K}}$ into the expression for divergence of the vector field in the toroidal coordinates

$$
\begin{aligned}
\vec{\nabla} \cdot \vec{V}=\frac{q}{a}[ & \frac{\partial V_{\eta}}{\partial \eta}+\frac{\partial V_{\xi}}{\partial \xi}+\frac{1}{\sinh \eta} \frac{\partial V_{\varphi}}{\partial \varphi}-2 V_{\xi} \frac{\sin \xi}{q} \\
& \left.+V_{\eta}\left(\frac{\cosh \eta}{\sinh \eta}-\frac{2 \sinh \eta}{q}\right)\right]
\end{aligned}
$$

we arrive at

$$
\frac{\partial}{\partial \eta} \ln \frac{f f^{\prime}}{\left(f^{2}+1\right)^{2}}=-\frac{2 m^{2}(\sinh \eta)^{2}-n^{2}}{m^{2}(\sinh \eta)^{2}+n^{2}} \frac{\cosh \eta}{\sinh \eta} .
$$

We take $m^{2}>n^{2}$ in Eq. (16). The integration yields

$$
\frac{1}{f^{2}+1}=\frac{2 k_{1}}{|m|\left(m^{2}-n^{2}\right)} \frac{\cosh \eta}{\left(\frac{n^{2}-m^{2}}{m^{2}}+\cosh ^{2} \eta\right)^{1 / 2}}+k_{2},
$$

with $k_{2}$ being the integration constant of the last integration. Imposing boundary conditions

$$
\vec{n} \rightarrow(0,0,1) \quad \text { or } \quad|u| \rightarrow \infty \quad \text { or } \quad f \rightarrow \infty \quad \text { as } \eta \rightarrow 0 \text {, }
$$

$$
\vec{n} \rightarrow(0,0,-1) \quad \text { or } \quad u \rightarrow 0 \quad \text { or } \quad f \rightarrow 0 \quad \text { as } \eta \rightarrow \infty \text {, }
$$

one gets

$$
f^{2}=\frac{\cosh \eta-\sqrt{n^{2} / m^{2}+\sinh ^{2} \eta}}{\sqrt{1+\left(m^{2} / n^{2}\right) \sinh ^{2} \eta}-\cosh \eta} .
$$

One observes that $f$ depends only on the ratio $\mathrm{m}^{2} / \mathrm{n}^{2}$, and that $f^{2} \geq 0$ for any value of $m^{2} / n^{2}$. Note that taking the limit $m \rightarrow n$ in (20) and using L'Hôpital's rule yields

$$
\lim _{m \rightarrow n} f^{2}=\frac{1}{\sinh ^{2} \eta}
$$


which gives for $m=n$ a solution

$$
u=\frac{e^{i m(\xi+\varphi)}}{\sinh \eta}
$$

In the special case of $m=n=1$, Eq. (22) reproduces the standard soliton solution [3,6]. Expression (22) can be written as a composite of the Hopf map together with the stereographic map: $\mathbb{R}^{3} \rightarrow S^{3}$ of degree 1 .
A formula,

$$
E \equiv \int d^{3} x \Theta_{00}=8^{3 / 4} \int d^{3} x \frac{\left(K_{i} \partial^{i} u^{*}\right)^{3 / 4}}{\left(1+|u|^{2}\right)^{3}},
$$

describes energy of the static configuration [3]. Inserting our solution into (23) we obtain an expression for the energy $E=E_{m, n}$ of the soliton configuration

$$
E_{m, n}=(2 \pi)^{2} 8 \times 2^{3 / 4} \int_{0}^{\infty} \frac{d \eta \sinh \eta}{(1+f)^{3}}\left(m^{2}+\frac{n^{2}}{(\sinh \eta)^{2}}\right)^{3 / 4} f^{3 / 2} f^{13 / 2}
$$

which after the $\eta$ integration yields

$$
E_{m, n}=(2 \pi)^{2} 4 \times 2^{1 / 4} \sqrt{|n||m|(|n|+|m|)} .
$$

We now turn to calculation of the Hopf numbers. Define functions $\Phi_{i}, i=1, \ldots, 4$, as follows:

$$
\begin{aligned}
\Phi_{\left\{{ }_{2}{ }_{2}\right\}}= & \left(\frac{\cosh \eta-\left(n^{2} / m^{2}+\sinh ^{2} \eta\right)^{1 / 2}}{(|m / n|-1)\left(n^{2} / m^{2}+\sinh ^{2} \eta\right)^{1 / 2}}\right)^{1 / 2} \\
& \times\left\{\begin{array}{c}
\cos m \xi \\
\sin m \xi
\end{array}\right\}, \\
\Phi_{\left\{4_{4}^{3}\right\}}= & \left(\frac{\left[1+\left(m^{2} / n^{2}\right) \sinh ^{2} \eta\right]^{1 / 2}-\cosh \eta}{(|m / n|-1)\left(n^{2} / m^{2}+\sinh ^{2} \eta\right)^{1 / 2}}\right)^{1 / 2} \\
& \times\left\{\begin{array}{c}
\cos n \varphi \\
-\sin n \varphi
\end{array}\right\} .
\end{aligned}
$$

They provide parametrization of $S^{3}$ and satisfy

$$
u=\frac{Z_{1}}{Z_{2}}=\frac{\Phi_{1}+i \Phi_{2}}{\Phi_{3}+i \Phi_{4}} \quad\left|Z_{1}\right|^{2}+\left|Z_{2}\right|^{2}=1
$$

or equivalently, for $\vec{n}$ defined from $u$ via relation (3),

$$
n_{i}=Z^{\dagger} \sigma_{i} Z ; \quad Z^{\dagger}=\left(Z_{1}^{*}, Z_{2}^{*}\right) ; \quad Z=\left(\begin{array}{c}
Z_{1} \\
Z_{2}
\end{array}\right) .
$$

Let, furthermore,

$$
A_{i}=\frac{i}{2}\left(Z^{\dagger} \partial_{i} Z-\partial_{i} Z^{\dagger} Z\right)
$$

be a vector potential for the two-form $H_{i j}=\partial_{i} A_{j}-$ $\partial_{j} A_{i}$. In terms of the toroidal coordinates the components of $\vec{A}$ are

$$
\begin{gathered}
A_{\eta}=q \partial_{\eta}\left(\Phi_{3} \Phi_{4}\right) ; \quad A_{\xi}=-m q\left(\Phi_{1}^{2}+\Phi_{2}^{2}\right) ; \\
A_{\varphi}=-\frac{n q}{\sinh \eta}\left(\Phi_{3}^{2}+\Phi_{4}^{2}\right) .
\end{gathered}
$$

Next, we calculate the vector function $\vec{B}=\vec{\nabla} \times \vec{A}$ (such that $B_{i}=\epsilon_{i j k} H_{j k} / 2$ ), using expression for the curl operator in toroidal coordinates and plugging it into the Hopf index defined as

$$
Q_{H}=\frac{1}{4 \pi^{2}} \int d^{3} x \vec{A} \cdot \vec{B}
$$

for which we now find

$$
Q_{H}=\frac{n m}{2}\left[\left.\left(\Phi_{1}^{2}+\Phi_{2}^{2}\right)^{2}\right|_{0} ^{\infty}-\left.\left(\Phi_{3}^{2}+\Phi_{4}^{2}\right)^{2}\right|_{0} ^{\infty}\right]=-n m \text {. }
$$

It is always possible to choose the Hopf index such that it is positive $Q_{H} \geq 0$. This amounts to the right choice of orientation, which determines the sign of $Q_{H}$.

Note that the following inequality,

$$
|m|+|n| \geq 2 \sqrt{|n||m|},
$$

always holds. Hence

$$
\begin{aligned}
\sqrt{|n||m|(|n|+|m|)} & \geq \sqrt{2}(|n||m|)^{3 / 4} \\
\rightarrow E_{m, n} & \geq(2 \pi)^{2} 4 \times 2^{3 / 4}\left|Q_{H}\right|^{3 / 4},
\end{aligned}
$$

which agrees with the lower bound result by [7] for the Faddeev-Skyrme model.

Notice that the solution obtained above implies that the vertex field $\vec{n}$ [see Eq. (3)] is given by

$$
\begin{aligned}
& n_{1}=\frac{2 f}{f^{2}+1} \cos (m \xi+n \varphi) ; \\
& n_{2}=\frac{2 f}{f^{2}+1} \sin (m \xi+n \varphi) ; \\
& n_{3}=\frac{f^{2}-1}{f^{2}+1} .
\end{aligned}
$$

Since $f$ is a function of $\eta$ only, one observes that the surfaces of constant $n_{3}$ are tori. In addition, on those surfaces, the lines of constant $n_{1}$ and $n_{2}$ wind around the tori with frequencies in the $\xi$ and $\varphi$ directions given by $m$ and $n$, respectively. One can check that $n_{3}$ falls monotonically from $n_{3}=1$ at $\eta=0$ to $n_{3}=-1$ at $\eta=\infty$. In addition, the bigger the ratio $m / n$ is, the faster it performs that flip. Therefore the size of our soliton decreases with the increase of $m / n$. 
The Hopf index of our solution can alternatively be calculated in the following way. The solution provides a mapping of the spatial $\mathbb{R}^{3}$ into $S^{3}$ defined by $\sum_{i=1}^{4} \Phi_{i}^{2}=$ 1. Then, Eqs. (3) and (28) provide the Hopf map $S^{3} \rightarrow$ $S^{2}$. The Hopf index is given by the linking number of the preimages, under the Hopf map, of any two points of $S^{2}$. Consider the point $\vec{n}=(0,0,-1)$ which corresponds in $\mathbb{R}^{3}$ to $\eta \rightarrow \infty$. For this value of $\eta, \Phi_{1}, \Phi_{2}$ go to zero, $\Phi_{3}=\cos n \varphi$, and $\Phi_{4}=-\sin n \varphi$. On $S^{3}$ we find the circle $\Phi_{3}^{2}+\Phi_{4}^{2}=\cos ^{2} n \varphi+\sin ^{2} n \varphi=1$ of radius 1 , and as $\varphi$ varies between 0 and $2 \pi$ the preimage wraps $|n|$ times around the $\varphi$ direction. Similarly, the preimage of $\vec{n}=(0,0,1)$ corresponds to $\eta=0$. For this value of $\eta$, $\Phi_{3}, \Phi_{4}$ go to zero, $\Phi_{1}=\cos m \xi$, and $\Phi_{2}=\sin m \xi$. On $S^{3}$ we find the circle $\Phi_{1}^{2}+\Phi_{2}^{2}=\cos ^{2} m \xi+\sin ^{2} m \xi=$ 1 of radius 1, and as $\xi$ varies between 0 and $2 \pi$ the preimage wraps $|m|$ times around the $\xi$ direction. Since these two circles intersect, the linking number is $|\mathrm{nm}|$. That is, indeed, the Hopf number calculated above.

Finally, let us mention that other models circumventing Derrick's theorem have been proposed previously in the literature [6,8]. A common feature is that, like the model considered in the present paper, the Lagrangians are nonpolynomial functions of the fields and their derivatives. The corresponding solutions have been constructed and in the special case of [6] a soliton with the unit Hopf charge was obtained. To our knowledge, an infinite number of soliton solutions to the field theoretical equations of motion with the Hopf charges bigger than unity were not obtained previously in the literature in an exact form.

L.A.F. and A.H.Z. are partially supported by $\mathrm{CNPq}$ (Brazil), and H. A. is partially supported by NSF (PHY9820663).

[1] L. Faddeev, in 40 Years in Mathematical Physics (World Scientific, Singapore, 1995).

[2] J. Gladikowski and M. Hellmund, Phys. Rev. D 56, 5194 (1997); L. Faddeev and A. Niemi, Nature (London) 387, 58 (1997); R. A. Battye and P. M. Sutcliffe, Phys. Rev. Lett. 81, 4798 (1998); J. Hietarinta and P. Salo, Phys. Lett. B 451, 60 (1999).

[3] H. Aratyn, L. A. Ferreira, and A. H. Zimerman, Phys. Lett. B 456, 162 (1999).

[4] O. Alvarez, L. A. Ferreira, and J. Sánchez Guillén, Nucl. Phys. B529, 689 (1998).

[5] L. A. Ferreira and E.E. Leite, Nucl. Phys. B547, 471 (1999).

[6] D. A. Nicole, J. Phys. G 4, 1363 (1978).

[7] A. F. Vakulenko and L. V. Kapitansky, Sov. Phys. Dokl. 24, 432 (1979).

[8] S. Deser, M. J. Duff, and C. J. Isham, Nucl. Phys. B114, 29 (1976). 